\title{
Validação da Recovery Assessment Scale (RAS) no Brasil para avaliar a capacidade de superação das pessoas com esquizofrenia
}

\author{
Validation of the Recovery Assessment Scale (RAS) \\ in Brazil in people with schizophrenia \\ Tiago R. Silva',2, Arthur A. Berberian”,3, Ary Gadelha', Cecília C. Villares', Larissa C. Martini', \\ Rodrigo A. Bressan?
}

\section{RESUMO}

Objetivo: Adaptar para o Brasil e investigar a confiabilidade e validade da Recovery Assessment Scale (RAS) em pessoas com esquizofrenia. Métodos: Etapa 1 - foi realizada tradução profissional para o português, adaptação e retrotradução da RAS. Etapa 2 - estudo-piloto em um grupo de 12 pessoas com esquizofrenia para garantir compreensão dos itens da escala. Etapa 3 - As pessoas com esquizofrenia $(N=104)$ foram submetidas à versão brasileira da RAS e a instrumentos de funcionalidade, qualidade de vida e sintomas para busca de evidências de validade. Resultados: Os resultados revelaram bons índices de consistência interna e de precisão teste e reteste dos instrumentos. Foram estabelecidas evidências de validade convergente entre a RAS e medidas de qualidade de vida $(r=0,58 ; p<0,001)$, funcionamento ocupacional ( $r=0,40 ; p<0,001)$, habilidades de vida independente $(r=0,24 ; p<$ $0,02)$, gravidade $(C G 1, r=-0,31 ; p<0,003)$ sintomas da esquizofrenia: PANSS total $(r=-0,21 ; p<$ $0,05)$, PANSS negativa $(r=-0,28 ; p<0.007)$, PANSS positiva $(r=-0.08 ; p=0,437)]$ e depressão [Calgary $(r=-0,27 ; p<0,01)]$. A análise fatorial exploratória revelou seis fatores, sendo quatro

\section{Palavras-chave}

Esquizofrenia, atitude, autonomia pessoal, qualidade de vida. destes similares a estudos prévios. Conclusão: A partir deste estudo, considerou-se que a palavra "superação" reflete melhor o conceito de "recovery". A versão brasileira da escala RAS é um instrumento válido e reprodutível para aferir a capacidade de "superação" das pessoas com esquizofrenia.

\section{ABSTRACT}

Objective: To adapt and investigate the validity reliability study of the Brazilian version of the Recovery Assessment Scale-RAS in people with schizophrenia. Methods: Stage 1 - professional translation to Portuguese, adaptation, and back-translation of the RAS; Stage 2 - RAS was presented to 12 outpatients with schizophrenia to evaluate if they would be able to understand and respond to the instrument; Stage 3 - patients with schizophrenia $(n=104)$ were assessed with the Brazilian version of the RAS, functional outcomes measures, quality of life and symptoms scales. Results: Results showed good internal consistency and retest reliability, and convergent validity between the RAS and quality of life measures $(r=0.58$;

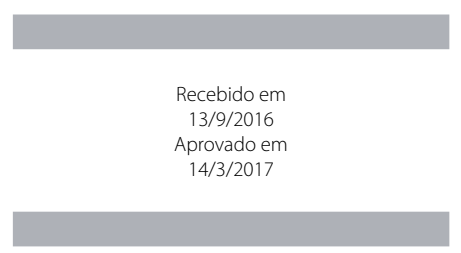

DOI: 10.1590/0047-2085000000144
1 Universidade Federal de São Paulo (Unifesp), Departamento de Psiquiatria, Programa de Esquizofrenia (Proesq). 2 Poliambulatório Uniamérica SCNSA.

3 Centro Universitário FIE0, Programa Stricto Sensu em Psicologia Educacional.

Endereço para correspondência: Rodrigo A. Bressan

Universidade Federal de São Paulo, Escola Paulista de Medicina, Departamento de Psiquiatria

Laboratório Interdisciplinar de Neurociências Clínicas

Edifício de Pesquisas II

Rua Pedro de Toledo, 669, $3^{\circ}$ andar fundos

04039-032 - São Paulo, SP, Brasil

E-mails: rodrigoabressan@gmail.com, arthur.berberian@gmail.com 


\section{Keywords}

Schizophrenia, attitude, personal autonomy, quality of life. $p<0.001)$, occupational functioning $(r=0.40 ; p<0.001)$, independent living skills $(r=0.24$; $p<0,02)$, functionality (CGI, $r=-0.31 ; p<0.003)$ and symptoms of schizophrenia, including PANSS total score total $(r=-0.21 ; p<0.05)$, PANSS negative $(r=-0.28 ; p<0.007)$, PANSS positive $(r=-0.08 ; p=0.48)$, and Calgary Depression Scale $(r=-0.27 ; p<0.01)$ ]. Exploratory factor analysis yielded six factors, four of these very similar to previous studies. Conclusion: This study suggests that the word "superação" ("overcome") better reflects the concept of "recovery" in Brazilian Portuguese. The Brazilian version of RAS is a valid and reliable instrument to evaluate the process of recovery in people with schizophrenia.

\section{INTRODUÇÃO}

Recovery é um processo profundo e subjetivo direcionado para mudança e estruturação da identidade de pessoas acometidas por doenças graves e crônicas, entre outros desafios. Esse processo possibilita que o indivíduo mantenha a esperança e estabeleça novas perspectivas de vida, aumentando sua participação no meio social',2.

Embora a esquizofrenia seja um transtorno psiquiátrico com altos índices de prejuízos funcionais ${ }^{3}$, dois terços dos indivíduos acometidos conseguem alcançar níveis satisfatórios de recovery ${ }^{4-6}$. Esse dado é determinante para a reformulação do conceito de incapacidade que gravita em torno das doenças mentais graves. Com efeito, propostas de tratamento psicossociais, baseadas em recovery, têm sido estruturadas em diferentes países ${ }^{7,8}$.

Por constituir um processo subjetivo, o construto recovery é influenciado por diferentes perspectivas, tais como valores, cultura e contexto, tornando difícil seu consenso entre pesquisadores e clínicos, e, consequentemente, de difícil mensuração objetiva ${ }^{9}$. Mesmo assim, dois são os enfoques genéricos dados na literatura para o termo: o da recuperação da doença e o de recuperação na doença ${ }^{10,11}$. O primeiro relaciona-se ao desfecho clínico, com base em parâmetros sintomáticos, melhora da funcionalidade e do ajuste social. Já o segundo relaciona-se a um processo contínuo que, embora possa variar de pessoa para pessoa, leva em consideração aspectos internos, tais como insight, integração, esperança, autonomia, superação e empoderamento, na sua avaliação. Além desses enfoques, três diferentes dimensões de recovery têm sido propostas $\left.{ }^{5}: 1\right)$ crença em si mesmo; 2) papel social, ter um papel ativo na sociedade; 3 ) relacionamento e interação com o meio. Tais dimensões constituem diretrizes fundamentais para a criação de instrumentos para avaliação desse construto ${ }^{12,13}$.

A Recovery Assessment Scale (RAS), desenvolvida por Corrigan et al. ${ }^{14}$, constitui uma das poucas escalas com desfechos claros e passíveis de serem utilizados em programas de intervenções. É uma escala autoaplicável que apresenta 41 itens em sua versão completa e 24 itens em sua versão reduzida e abrange cinco diferentes dimensões: 1) autoconfiança e esperança; 2) vontade de pedir ajuda; 3) objetivos pessoais e perspectiva de melhora; 4) confiança nos outros e 5) não se deixar dominar pelos sintomas. Ela tem demonstrado parâmetros psicométricos adequados e é a escala mais utilizada em diferentes contextos de saúde mental no mundo ${ }^{1421}$.

Apesar de ser relevante tanto para a realização de estudos como para a prática clínica, a RAS ainda não foi validada para uso no Brasil. Além disso, de modo geral, há carência de instrumentos padronizados para construtos como esse e com características psicométricas estabelecidas, que garantam seu adequado uso no país. Nesse sentido, a tradução, a adaptação e a validação de instrumentos que aferem recovery podem constituir um importante recurso para a área de saúde mental em contexto nacional, além de permitir o pareamento dessas características com estudos internacionais.

Dessa maneira, o presente estudo realizou a tradução, a retrotradução e a validação da RAS para a população brasileira. Mais especificamente, buscou-se avaliar se a versão traduzida e adaptada possui boa consistência interna, precisão teste-reteste, evidências de validade baseada na relação com outras variáveis e estrutura fatorial condizente ao construto.

\section{MÉTODOS}

\section{Desenho do estudo}

O estudo foi conduzido em três etapas: Etapa 1 - foi realizada tradução profissional para o português, adaptação e retrotradução da RAS; Etapa 2 - estudo-piloto em um grupo de 12 pessoas com esquizofrenia para garantir compreensão dos itens da escala; Etapa 3 - Estudo dos parâmetros psicométricos com uma amostra de 104 pacientes diagnosticados com esquizofrenia, estáveis, segundo o DSM-IV22, com idade entre 16 e 68 anos. Na terceira etapa foi feito um estudo de precisão da RAS por meio da avaliação da consistência interna e da confiabilidade teste e reteste com 45 pacientes reavaliados 15 dias após a primeira testagem. Também na terceira etapa os pacientes foram avaliados com uma série de instrumentos que aferem variáveis correlatas, tais como sintomas, qualidade de vida e funcionamento geral para avaliar a validade convergente da escala. Este estudo foi aprovado pelo Comitê de Ética da Universidade Federal de São 
Paulo (Unifesp) e todos os participantes aceitaram participar do estudo após serem informados extensamente sobre os procedimentos e assinaram o termo de consentimento livre e esclarecido.

Para a confirmação do diagnóstico de esquizofrenia, todos os participantes foram submetidos à Entrevista Semiestruturada para o Diagnostic and Statistical Manual of Mental Disorders, 4a ed. (SCID-IV) ${ }^{23}$. Os pacientes estavam sob tratamento com antipsicóticos de segunda geração em doses estáveis por pelo menos quatro semanas prévias à avaliação.

\section{Recovery Assessment Scale (RAS)}

As cinco dimensões da $\operatorname{RAS}^{14}$ são apresentadas em uma escala autoaplicável, sendo os itens pontuados por escala Likert de 1 a 5 (discordo plenamente $=1$; discordo $=2$; não tenho certeza $=3$; concordo $=4$ e concordo plenamente $=5$ ). Quanto maior a pontuação, melhor seria seu nível de enfrentamento da doença, utilização de estratégias e maior capacidade de superação. No estudo original, foi demonstrado que a versão reduzida, composta por 24 itens, é suficiente para aferir o processo de recovery ${ }^{7,24}$. Essa versão reduzida com 24 itens foi utilizada no presente estudo neste estudo, juntamente com a aplicação e validação da versão completa.

A tradução e a adaptação transcultural da RAS original foram realizadas de forma padronizada, de acordo com a seguinte sequência: 1. Tradução inicial: os itens da versão em inglês da RAS foram traduzidos conceitualmente para a língua portuguesa utilizada no Brasil por um profissional de saúde, bilíngue, brasileiro e ciente do objetivo desta pesquisa. 2. A tradução inicial foi retrotraduzida (back-translation) para o inglês por um tradutor bilíngue, independente, ciente do objetivo da pesquisa. Posteriormente, a retrotradução foi comparada com o instrumento original, indicando a equivalência semântica e reconciliação dos itens traduzidos. Para a realização da equivalência idiomática (expressões equivalentes não encontradas) e equivalência conceitual (validade do conceito explorado), alguns termos foram substituídos por outros, similares, pelo fato de os itens originais não se enquadrarem no linguajar habitual da população brasileira. Como, por exemplo, a frase "É importante ter muitos amigos" sendo substituída por "É importante ter vários amigos", como também a frase "Tenho minha própria maneira de como ficar e permanecer bem" sendo substituída por "Tenho minha própria maneira de como melhorar e ficar bem".

\section{Instrumentos de funcionalidade}

\section{WHOQOL-Brief}

Instrumento de avaliação de Qualidade de Vida (WHOQOL-Brief/OMS)25: é um instrumento estruturado, autoaplicável, com 26 questões distribuídas em quatro domínios: relações sociais, psicológico, físico e meio ambiente.

\section{ILSS-SR}

Inventário de Habilidades de Vida Independente (ILSS-SR) ${ }^{26}$ : avalia 10 domínios: aparência e vestuário, higiene pessoal, cuidado com os objetos pessoais, preparo e armazenamento dos alimentos, saúde, administração do dinheiro, transporte, lazer, emprego e manutenção do trabalho.

\section{SAOF}

Questionário de Autoavaliação do Funcionamento Ocupacional (SAOF): é um instrumento estruturado, autoaplicável, composto de 32 itens, com o objetivo de avaliar a percepção do paciente sobre seu funcionamento ocupacional nos seguintes papéis: estudante, amigo, familiar, hobby e sustento do lar.

\section{Instrumentos para avaliação clínica}

Todos os participantes foram submetidos à Escala das Síndromes Positivas e Negativas (PANSS) ${ }^{27}$. Essa medida inclui 30 itens: sete de sintomas positivos, sete itens de sintomas negativos e dezesseis de psicopatologia em geral. Os sintomas são mensurados por uma escala likert que varia de 1 a 7 , sendo a pontuação 1 ausência de sintomas e a 7 presença severa ${ }^{28}$

Para a avaliação de funcionamento global, foi utilizada a Avaliação Global de Funcionamento (GAF) ${ }^{29}$ e para gravidade geral, a Impressão Clínica Global (CGl) ${ }^{30}$. A primeira escala recebe uma pontuação de 0 a 100 para o nível de funcionalidade. A segunda é utilizada para a avaliação da gravidade global do paciente, considerando-se frequência e intensidade dos sintomas. Seu escore varia entre 1 (normal, não doente) e 7 (extremamente doente). Para avaliar os sintomas depressivos na esquizofrenia foi utilizada a Escala Calgary de Depressão para Esquizofrenia (ECDE) ${ }^{31}$ : escala semiestruturada que avalia sintomas depressivos em esquizofrenia, composta por nove itens, todos definidos de acordo com um critério operacional que vai de zero a três. A escala abrange o período das últimas duas semanas e deve ser aplicada numa entrevista diretiva por examinadores.

\section{Procedimentos}

No primeiro momento, os pacientes foram avaliados por dois psiquiatras treinados na aplicação do SCID, para elegibilidade no estudo. Nesta etapa os pacientes foram avaliados com a PANSS, Escala Calgary de Depressão, WHOQOL-Brief. No segundo momento, o pesquisador realizou a aplicação da RAS e dos instrumentos de funcionalidade. Os entrevistados foram orientados sobre a proposta da escala e eram acompanhados pelo pesquisador durante a aplicação, a fim de garantir um melhor entendimento dos itens e sanar possíveis dúvidas em relação à escala. Para o estudo de precisão teste e reteste, 45 pacientes foram recrutados e reavaliados após o período de tempo de 15 dias após a primeira testagem. 


\section{Análise estatística}

As análises estatísticas foram realizadas com o Pacote Estatístico para Ciências Sociais (SPSS), versão 18.0. A análise descritiva, incluindo média, frequências e desvios-padrão, foi calculada para todos os dados sociodemográficos. Cinco participantes apresentaram afastamento ou inconsistência do padrão (outliers). Eles foram eliminados do grupo de pacientes por terem sido considerados um possível erro de execução na tarefa, pois essa variabilidade não pareceu ser algo inerente da amostra ou um padrão esperado na população de pacientes. Assim, obteve-se um total de 99 participantes. Distribuição dos dados foi verificada por meio de Skewness, Kurtosis e pelo teste Kolmogorov-Smirnov.

Para estudos de precisão, a estabilidade da escala foi verificada pelo uso do coeficiente de correlação de Pearon por teste-reteste, já para consistência interna dos itens, o alfa de Cronbach. Para evidências de validade baseadas na relação com outras variáveis (critério concorrente), também foram utilizadas correlações de Pearson entre a RAS e as demais medidas de funcionalidade e de sintomas. A análise de componentes principais com rotação oblíqua (varimax) foi utilizada para evidências de validade baseada na estrutura internada escala. Fatores com autovalores (eigenvalues) maiores do que 1 foram extraídos e cargas fatoriais acima de 0,40 foram consideradas como relevantes.

\section{RESULTADOS}

\section{Etapa 1 - Estudo-piloto}

Com o objetivo de qualificar o processo de adaptação cultural e atingir consenso dos conceitos abordados pela RAS, foi realizado um estudo-piloto em um grupo focal, com 12 pacientes com esquizofrenia.

Os participantes responderam coletivamente à escala, item a item, com a presença e o acompanhamento do avaliador e, em seguida, expuseram suas impressões sobre a escala e os itens respondidos. A partir da transcrição sobre a impressão dos participantes, foi decidido realizar uma formatação visual da escala, colocando em cada item as opções de resposta com sua respectiva numeração, para facilitar a visualização e o melhor preenchimento pelos participantes. Outro aspecto incluído a partir do grupo-piloto foi a elaboração de um enunciado explicativo sobre a escala, com uma breve definição do tema e a substituição do termo em inglês recovery, que foi trocado pela palavra recuperação em português. Após amplas discussões baseadas na vasta experiência dos pesquisadores (CCV) com o tema e avaliação dos dados do projeto-piloto, foi decidido que a palavra "superação" expressa melhor o conceito original de recovery.

\section{Etapa 2 - Estudo dos parâmetros psicométricos}

A Tabela 1 apresenta os dados sociodemográficos da amostra. Houve um predomínio de indivíduos do sexo masculino
$(68,3 \%)$ em relação ao sexo feminino $(31,7 \%)$. Os participantes tinham entre 16 e 68 anos de idade, com predominância de 57,7\% na faixa de 29 e 48 anos. Em relação ao estado civil, 73,1\% eram solteiros e 11,5\% viviam com um(a) companheiro(a). No item "com quem mora", 58,7\% moravam com os pais e 2,9\% moravam sozinhos. Quanto ao aspecto da "ocupação atual", 62,5\% eram desempregados e $17,3 \%$ tinham algum emprego registrado e não registrado. Em relação ao número de internações psiquiátricas, 81,7\% tiveram de 0 a 5 internações. 0 tempo de doença apresentou valores semelhantes com a faixa etária, respectivamente de 6 a 20 anos de doença (37,5\%) e de 21 a 29 anos (38,5\%).

Tabela 1. Dados demográficos dos participantes com esquizofrenia $(\mathrm{N}=99)$

\begin{tabular}{|c|c|c|}
\hline Domínio & $\%$ & Média (DP) \\
\hline Idade (em anos) & & $37,40(11,05)$ \\
\hline Escolaridade (em anos) & & $5,58(1,81)$ \\
\hline Número de internações & & $2,13(3,13)$ \\
\hline Duração de doença (em anos) & & $6,08(5,05)$ \\
\hline Idade de início & & $22,78(6,86)$ \\
\hline \multicolumn{3}{|l|}{ Com quem mora } \\
\hline Sozinho & 2,9 & \\
\hline Pais & 58,7 & \\
\hline Companheiro & 13,5 & \\
\hline Outros & 16,3 & \\
\hline Não informou & 8,6 & \\
\hline \multicolumn{3}{|l|}{ Sexo } \\
\hline Masculino & 68,3 & \\
\hline Feminino & 31,7 & \\
\hline \multicolumn{3}{|l|}{ Ocupação atual } \\
\hline Desempregado & 62,5 & \\
\hline Empregado & 24,0 & \\
\hline Estudante & 1,9 & \\
\hline Não informou & 11,6 & \\
\hline \multicolumn{3}{|l|}{ Estado civil } \\
\hline Solteiro & 73,0 & \\
\hline Companheiro & 11,5 & \\
\hline Separado & 5,8 & \\
\hline Viúvo & 1,0 & \\
\hline Não informou & 8,7 & \\
\hline \multicolumn{3}{|l|}{ PANSS } \\
\hline Sintomas positivos & & $13,00(4,83)$ \\
\hline Sintomas negativos & & $16,43(5,45)$ \\
\hline Psicopatologia geral & & $28,97(7,20)$ \\
\hline Escore total & & $57,91(14,13)$ \\
\hline ECDE & & $2,39(3,46)$ \\
\hline GAF & & $50,85(12,96)$ \\
\hline CGI & & $3,78(1,00)$ \\
\hline
\end{tabular}

ECDE: Escala Calgary de Depressão para Esquizofrenia; GAF: Avaliação Global de Funcionamento; CGI: Impressão Clínica Global; DP: desvio-padrão. 


\section{Estudo de precisão}

O estudo de precisão demonstrou alfa de Cronbach de 0,937 para a versão completa com 41 itens, e de 0,878 para a versão reduzida com 24 itens. A análise de correlação teste-reteste apresentou valor $r=0,70$ e significância de $p<0,001$. O alfa de Cronbach para o reteste $(N=45)$ foi de 0,94.

\section{Evidências de validade baseada na relação com outras variáveis}

A validade concorrente foi examinada pela comparação do escore global da RAS versão completa com as escalas SAOF, ILSS, PANSS, Calgary, GAF, CGI, WHOQOl-brief.

As correlações demonstraram-se significantes com todas as escalas: WHOQOL $(r=0,58 ; p<0,001)$, SAOF $(r=0,40 ; p<$ $0,001)$, GAF $(r=0,27 ; p<0,009)$, ILSS $(r=0,24 ; p<0,02)$, CGI $(r=-0,31 ; p<0,003)$, CALGARY $(r=-0,27 ; p<0,01)$, PANSS total $(r=-0,21 ; p<0,05)$, PANSS negativa $(r=-0,28 ; p<0,007)$ e PANSS positiva $(r=-0,08 ; p=0,437)$.

\section{Análise da estrutura interna da RAS versão reduzida}

O estudo da validade fatorial da RAS foi feito com a versão reduzida RAS com 24 itens, com base em estudos prévios (Chiba et al., 2010; Corrigan et al., 2001; McNaught et al., 2007), e realizado por meio da análise fatorial exploratória (AFE) com o método maximum likelihood com rotação ortogonal varimax, apresentando Kaiser-Meyer-Olkin (KMO) de 0,79. O Teste de Esfericidade de Bartlett, usado para examinar a hipótese de que as variáveis não sejam correlacionadas na população, foi significativo $\left(x^{2}=684,78, g l=276, p<0,001\right)$.

A AFE produziu seis diferentes fatores, conforme a Tabela 2. Alguns itens apresentaram carga fatorial superior a 0,40 e, portanto, compuseram mais de um fator. O Fator 1 explicou 28,82\% da variância (7 itens), o fator 2 teve 5,12\% da variância explicada (6 itens), o fator 3 apresentou 5,54\% (5 itens), o fator 4 teve $4,77 \%$ ( 3 itens), fator 5 teve $6,04 \%$ ( 3 itens) e o fator 6 apresentou 3,40\% (2 itens). Apenas o item 37 não apresentou carga fatorial superior ao critério estipulado.

Um estudo de correlações entre as diferentes dimensões das RAS com os demais instrumentos também foi conduzido para uma melhor compreensão das relações de cada dimensão da superação com funcionalidade e sintomas. Os escores de qualidade de vida da WHOQOL e da SAOF apresentaram correlações de fraca a moderada $(0,24-0,42)$. A Tabela 3 sumaria os coeficientes de correlação.

\section{DISCUSSÃO}

O presente estudo descreve a tradução, a adaptação e a validação da versão brasileira da RAS. Os resultados retratam quatro aspectos principais. Primeiro, as avaliações dos itens e instruções da versão traduzida, feitas no estudo-piloto, demonstraram que os pacientes puderam compreender a escala e responder às questões adequadamente. Segundo, além de alta consistência interna tanto para a versão completa (41 itens), como para a versão reduzida (24 itens), a análise de correlação entre o teste e o reteste mostrou-se satisfatória. Terceiro, a RAS apresentou magnitudes correlacionais moderadas com uma medida de qualidade de vida e outra de funcionalidade ocupacional. Finalmente, a estrutura interna da RAS organizou-se em seis diferentes fatores, cada um representando as diferentes facetas envolvidas no construto.

A tradução literal da palavra recovery para a língua portuguesa é "recuperação", que significa reconquista da saúde, do bem-estar e do funcionamento social e laboral ${ }^{37}$. Neste estudo, o uso da palavra recuperação estava consoante com o relato das pessoas com esquizofrenia sobre estar num processo contínuo de recuperação da doença e não em um estado permanente de reconquista do estado de saúde. Assim, considerando também os processos subjetivos de mudança e estruturação da identidade, foi proposto o termo "superação". Conforme Miranda et al.36 "superação", no enquadre da saúde mental, é um termo usado para descrever um conceito que tem reivindicado a importância de que se reconheçam e validem os processos de superação na doença que as levam a viver melhor apesar da doença ou dos sintomas desta. A autora complementa que as práticas orientadas pela superação abrangem a perspectiva da pessoa em tratamento, consideram suas potencialidades e estratégias de enfrentamento e, ao se orientarem para o futuro, incentivam a corresponsabilidade e uma postura de esperança no processo de conviver com a doença e superar as adversidades impostas por ela ${ }^{36}$. Dessa forma, a palavra "superação" parece refletir melhor o conceito original, pois um indivíduo pode manter sintomas psicóticos ativos ao longo de sua vida e mesmo assim atingir a "superação" por ter autonomia e esperança, além de estabelecer boas relações sociais e ter um papel ativo na sociedade. Em oposição, um indivíduo pode ter se recuperado em relação ao processo de doença (remissão de sintomas), mas não atingir a "superação", quando não consegue ter relações sociais a contento e um papel na sociedade, por ter baixa autoestima, esperança e depender de outras pessoas para viver. "Superação" nos parece uma palavra mais positiva que transcende as questões ligadas à doença e empodera as pessoas focarem na sua vida muito além do âmbito clínico.

No que tange à precisão das medidas, os adequados resultados permitem interpretar que a versão brasileira está relativamente livre de vieses determinados por particularidades de itens específicos e a precisão teste-reteste demonstra ausência de flutuações significativas nos resultados da RAS em diferentes momentos. Esses dados são equiparados aos estudos de validação da RAS no âmbito internacional7,14,16,17. Desse modo, os índices de precisão foram satisfatórios, sugerindo boa consistência interna e boa precisão teste-reteste. 
Tabela 2. Matriz dos componentes rotacionados de todas os itens da RAS

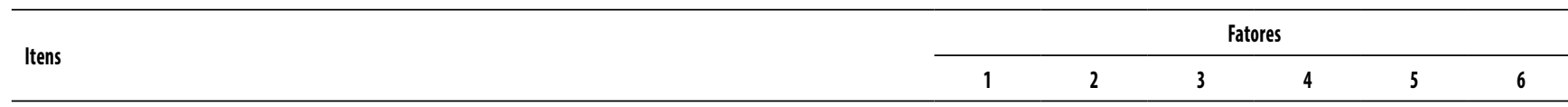

Fator 1 - Objetivos pessoais e perspectiva de melhora

01 - Tenho vontade de ter sucesso (GO)

0,67

03 - Tenho objetivos na minha vida que desejo alcançar (GO)

0,57

0,56

0,72

0,42

0,54

15 - Eu gosto de mim mesmo (PE)

05 - Tenho um propósito de vida (G0)
0,38

0,31

0,30

$\begin{array}{ccc} & 0,67 & \\ & 0,43 & 0,32 \\ & 0,64 & \\ 0,30 & 0,60 \\ & 0,54 \\ & 0,58\end{array}$

0,41

Fator 3 - Autoconfiança e esperança

16 - Se as pessoas realmente me conhecessem, gostariam de mim (PE

0,60

0,52

0,31

0,42
0,40

0,70

0,34

0,51

25 - Continuo tendo novos interesses (PE)

Fator 4 - Não se dominar pelos sintomas

11 - 0 medo não me impede de viver da maneira que eu quero (PE)

0,46

28 - Meus sintomas interferem cada vez menos na minha vida (NO)

0,72

29 - Meus sintomas parecem durar menos à medida que eles ocorrem (NO)

0,76

\section{Fator 5 - Vontade de pedir ajuda}

27 - Enfrentar a minha doença mental é, sem dúvida, o maior foco da minha vida (N0)

31 - Estou com vontade de pedir ajuda (WI)

0,71

\section{Fator 6 - Confiança nos outros}

37 - Tenho pessoas com quem posso contar (RE)

40 - É importante ter vários amigos (RE)

Autovalores

\% variância explicada

Alfa de Cronbach do fator

\begin{tabular}{cccccc} 
& 0,32 & 0,32 & & & 0,34 \\
7,38 & 1,91 & 1,90 & 1,65 & 1,60 & 0,83 \\
30,74 & 7,97 & 7,11 & 6,89 & 6,25 & 5,15 \\
0,83 & 0,70 & 0,75 & 0,73 & 0,57 & 0,60 \\
\hline
\end{tabular}

G0: objetivos pessoais e perspectiva de melhora; PE: autoconfiança e esperança; RE: confiança nos outros; WI: vontade de pedir ajuda; N0: não se dominar pelos sintomas.

Números em negrito indicam cargas fatoriais maiores que 0,40 .

Tabela 3. Correlação de Pearson da RAS com escalas utilizadas $(N=99)$

\begin{tabular}{|c|c|c|c|c|c|c|c|c|c|c|}
\hline Fatores RAS & CGI & GAF & $\begin{array}{l}\text { Escore Total } \\
\text { PANSS }\end{array}$ & $\begin{array}{l}\text { Escore Escala } \\
\text { Geral }\end{array}$ & $\begin{array}{l}\text { Escore Escala } \\
\text { Negativa }\end{array}$ & $\begin{array}{c}\text { Escore Escala } \\
\text { Positivo }\end{array}$ & Escore Calgary & $\begin{array}{l}\text { WHO-QOI } \\
\text { resultado total }\end{array}$ & $\begin{array}{l}\text { ILSS resultado } \\
\text { total }\end{array}$ & $\begin{array}{c}\text { SAOF resultado } \\
\text { total final }\end{array}$ \\
\hline Fator 1 & $-0,15$ & 0,17 & $-0,08$ & $-0,09$ & $-0,17$ & 0,33 & $-0,18$ & $0,30^{*}$ & 0,18 & 0,12 \\
\hline Fator 2 & $-0,14$ & 0,17 & $-0,14$ & $-0,11$ & $-0,18$ & 0,05 & 0,08 & $0,38^{* * *}$ & 0,19 & $0,31^{*}$ \\
\hline Fator 3 & $-0,21$ & 0,17 & $-0,6$ & 0,01 & $-0,04$ & $-0,15$ & $-0,17$ & $0,27^{* *}$ & $-0,10$ & 0,18 \\
\hline Fator 4 & $-0,25$ & 0,05 & $-0,09$ & 0,50 & $-0,03$ & $-0,20$ & 0,02 & $0,42^{* * *}$ & 0,10 & $0,24^{* *}$ \\
\hline Fator 5 & $-0,11$ & 0,18 & 0,02 & 0,90 & $-0,13$ & 0,11 & 0,19 & 0,08 & $0,03^{*}$ & $0,21^{*}$ \\
\hline Fator 6 & $-0,09$ & 0,05 & 0,01 & 0,80 & 0,09 & $-0,09$ & $-0,02$ & 0,12 & 0,09 & $-0,50$ \\
\hline
\end{tabular}

${ }^{*} p<0,05 ;{ }^{* *} p<0,01 ;{ }^{* * *} p<0,001$. 
Quanto à busca por evidências de validade, baseada na relação com outras variáveis, a versão brasileira da RAS demonstrou nível significativo e correlação moderada com a escala WHOQOL-brief de qualidade de vida. Essa correlação pode ser explicada por Young e Ensing ${ }^{32}$ e Deegan ${ }^{33}$, que referem aspectos de mudanças positivas tanto no âmbito pessoal como social na vida do portador, contribuindo para uma maior satisfação com sua própria vida.

Em relação às escalas GAF e ILSS, a correlação foi significativa, mas mostrou-se baixa. Tal fato pode estar relacionado com o contexto familiar dos entrevistados. No grupo estudado, $58,7 \%$ moravam com os pais e $13,5 \%$ com o(a) companheiro(a), significando que $72,2 \%$ da amostra poderia ser composta por pessoas algo dependentes para algumas atividades de vida diária e estarem desempregadas (62,5\%). A correlação da RAS com as escalas PANSS e Calgary foi negativa e mostrou-se baixa, necessitando maiores estudos entre a RAS brasileira com essas escalas. Outros estudos de validação da RAS confirmam a relação já observada que, quanto maior a intensidade dos sintomas negativos e positivos vivenciados pelo portador, menor seria sua capacidade de superação, desempenho de papéis ocupacionais e desenvolvimento de uma perspectiva de vida mais positiva $7,14,16$.

A análise fatorial produziu seis fatores na amostra total. Os Fatores 1, 3, 4 e 6 foram bastante comparáveis aos fatores de estudos prévios ${ }^{14,16,18}$. O Fator 1 , como nos estudos anteriormente citados, agrupou itens relacionados a objetivos pessoais e perspectiva de melhora com itens de autoconfiança e esperança, possivelmente pelo fato de os participantes relacionarem objetivos e sucesso com esperança. O Fator 2 estabeleceu-se como uma dimensão com itens de diferentes dimensões, mas quatro dos seis itens que o compõem parecem envolver uma ideia de controle interior, sendo eles: 1) "tenho minha própria maneira de como ficar", que é classificado como um item de objetivos pessoais e de perspectiva de melhora, 2) "consigo lidar com o que acontece na minha vida"; 3) "consigo lidar com o estresse", estes últimos classificados como sendo de autoconfiança; e 4) "eu sei quando pedir ajuda", este classificado como sendo de vontade de pedir ajuda, mas com ênfase em se saber quando fazer algo. Este último item alcançou carga fatorial de 0,60. Todos os itens do Fator 3 eram sobre "autoconfiança e esperança" e os dois itens com maiores cargas fatoriais do Fator 4 são sobre "não se dominar pelos sintomas". O Fator 5 apresentou heterogeneidade de itens do ponto de vista teórico, sendo o mais discrepante com os estudos prévios, com um item de "confiança nos outros", "vontade de pedir ajuda" e "objetivos pessoais e perspectiva de melhora". O Fator 6 também uniu três fatores relacionados à confiança nos outros, sendo que um obteve carga fatorial inferior a 0,40 .

Como limitação do estudo, ressalta-se que o aspecto cultural da amostra brasileira pode refletir nas diferentes formas das pessoas se relacionarem com a doença, sintomas, relações familiares e, consequentemente, sobre as estratégias de apoio que desenvolvem ou consideram como importantes. Outro aspecto a ser ressaltado sobre a amostra está relacionado à gravidade da doença, à baixa crítica ou ao nível educacional de alguns portadores sobre sua própria doença, sendo considerado um possível viés de interpretação e resposta. Verificou-se que a presença do entrevistador no momento da aplicação da RAS foi uma condição facilitadora para esclarecimentos e dúvidas que surgiram por parte dos entrevistados.

Destaca-se o potencial avaliativo da RAS sobre a capacidade de superação dos portadores, constituindo uma foto do momento de vida e perspectiva que eles possuem. Faz-se necessário, então, testar se níveis elevados dos fatores da RAS poderiam predizer melhor resposta a intervenções psicossociais aos portadores de esquizofrenia. A relação entre a capacidade de superação e o desempenho funcional encontra-se ainda incipiente, necessitando-se de mais estudos de intervenção para buscarmos maiores evidências.

\section{CONCLUSÃO}

A partir dos dados deste estudo, fica claro que a palavra "superação" parece mais apropriada para refletir o conceito original do termo em inglês "recovery". Considera-se que a versão brasileira da RAS é um instrumento com acúmulo de dois tipos de evidências de validade (convergente e fatorial) e de precisão (teste-reteste e de consistência interna) para aferir as dimensões da capacidade de superação dos portadores de esquizofrenia, tanto em sua versão reduzida como em sua versão completa.

Por se tratar de uma medida processual, ligada a aspectos intrínsecos sobre a experiência de portadores de esquizofrenia ${ }^{33-35}$, a versão brasileira da RAS pode ser indicada como protocolo para serviços de saúde mental, orientados ao processo de desenvolvimento da capacidade de superação das pessoas com transtornos mentais.

O conceito de "superação" tem um papel central no tratamento de pessoas com transtornos mentais e representa uma grande evolução para a prática clínica. No entanto, ainda não foi inteiramente incorporado pelos profissionais de saúde mental. Este estudo fez uma adaptação cientificamente rigorosa da escala RAS para uso no Brasil e espera-se que tenha um importante papel tanto no desenvolvimento de pesquisas como ampla implementação do conceito de "superação" na prática no trabalho clínico com pessoas com transtorno mental.

\section{CONTRIBUIÇÕES INDIVIDUAIS}

Tiago R. Silva - Elaborou o estudo, foi responsável pela coleta de dados, pela tabulação e análise dos dados, bem como pela escrita deste artigo. 
Arthur A. Berberian - Analisou e interpretou os dados e participou da elaboração do estudo e da escrita deste artigo.

Ary Gadelha - Contribuiu com a orientação no estudo, com os dados sociodemográficos e as avaliações psiquiátricas, PANSS, GAF, CGl e Calgary da amostra.

Cecília C. Villares - Contribuiu na co-orientação do estudo, principalmente nos aspectos conceituais de "recovery".

Larissa C. Martini - Contribuiu com orientações na coleta dos dados da escala ILSS e auxiliou na aplicação das escalas de funcionalidade.

Rodrigo A. Bressan - Foi o orientador geral durante toda a duração do estudo, tanto metodológica como conceitualmente, bem como na escrita deste artigo.

\section{CONFLITOS DE INTERESSE}

Os autores declaram que não possuem qualquer conflito de interesse para este manuscrito.

\section{AGRADECIMENTOS}

Gostaríamos de agradecer a todos os pesquisadores e funcionários do Programa de Esquizofrenia (Proesq) que colaboraram, de alguma maneira, para a pesquisa e aos participantes da amostra, que cederam seu tempo, suas experiências e puderam nos ensinar um pouco mais sobre seus processos de superação da doença.

\section{REFERÊNCIAS}

1. Ahern L, Fisher D. Personal Assistance in Community Existence: Recovery at Your Own Pace. Lawrence, MA: National Empowerment Center, 1999.

2. Duarte T. Recovery da doença mental: uma visão para os sistemas e serviços de saúde mental. Aná Psicológica. 2007;1(25):127-33.

3. Fett AK, Viechtbauer W, Dominguez MD, Penn DL, van Os J, Krabbendam L. The relationship between neurocognition and social cognition with functional outcomes in schizophrenia: a meta-analysis. Neurosci Biobehav Rev. 2011;35(3):573-88.

4. Anthony WA. The need for recovery-compatible evidence based practices. Mental Health Weekly. 2001. p. 5.

5. Schiff AC. Recovery and mental illness: analysis and personal reflections. Psychiatr Rehabil J. 2004 Winter;27(3):212-8.

6. Sartorius N. Twenty-five years of WHO-Coordinated Activities concerned with schizophrenia. In: Hooer K, Harrison G, Janca A, Sartorius N, editors. Recovery from Schizophrenia: na International Perspective. New York: Oxford University Press, 2007.

7. Corrigan PW, Phelan SM. Social support and recovery in people with serious mental illnesses. Community Ment Health J. 2004;40(6):513-23.

8. Yanos PT, Roe D, Lysaker PH. The Impact of IIIness Identity on Recovery from Severe Mental IIness. Am J Psychiatr Rehabil. 2010;13(2):73-93.

9. Jacobson N, Greenley D. What is recovery? What is recovery? A conceptual model and explication. Psychiatr Serv. 2001;52(4):482-5.

10. Liberman RP, Kopelowicz A. Recovery from schizophrenia: a concept in search of research. Psychiatr Serv. 2005;56(6):735-42.

11. Essock $S$, Sederer L. Understanding and measuring recovery. Schizophr Bull. 2009;35(2):279-81.
12. Almeida PF, Escorel S. 0 desafio da produção de indicadores para avaliação de serviços em saúde mental: um estudo de caso do Centro de Atenção Psicossocial Rubens Corrêa/RJ; Tese de Mestrado em Ciências da Saúde Pública pela Escola Nacional de Saúde Pública da Fundação Oswaldo Cruz - ENSP/Fiocruz; 2002.

13. Alsop R, Heinsohn N. Measuring Empowerment in Practice: Structuring Analysis and Framing Indicators, World Bank, Washington DC; 2005.

14. Corrigan PW, Giffort D, Rashid F, Leary M, Okeke I. Recovery as a psychological construct. Community Ment Health J. 1999;35(3):231-9.

15. Corrigan PW. Impact of consumer-operated services on empowerment and recovery of people with psychiatric disabilities. Psychiatr Serv. 2006;57(10):1493-6.

16. McNaught M, Caputi P, Oades LG, Deane FP. Testing the validity of the Recovery Assessment Scale using an Australian sample. Aust N Z J Psychiatry. 2007;41(5):450-7.

17. Chiba R, Miyamoto Y, Kawakami N. Reliability and validity of the Japanese version of the Recovery Assessment Scale (RAS) for people with chronic mental illness: scale development. Int J Nurs Stud. 2010;47(3):314-22.

18. Barbic S, Krupa T, Armstrong I. A randomized controlled trial of the effectiveness of a modified recovery workbook program: preliminary findings. Psychiatr Serv. 2009;60(4):491-7.

19. Mueser KT, Meyer PS, Penn DL, Clancy R, Clancy DM, Salyers MP. The IIIness Management and Recovery program: rationale, development, and preliminary findings. Schizophr Bull. 2006;32 Suppl 1:\$32-43.

20. Oades, L, Deane F, Crowe T, Lambert WG, Kavanagh D, Lloyd C. Collaborative recovery: an integrative model for working with individuals who experience chronic and recurring mental illness. Australas Psychiatry. 2005;13(3):279-84.

21. Hendryx M, Green CA, Perrin NA. Social support, activities, and recovery from serious mental illness: STARS study findings. J Behav Health Serv Res. 2009;36(3):320-9.

22. American Psychiatric Associaton. Avaliação multiaxial. In: Manual diagnóstico e estatístico de transtornos mentais (DSM-IV-TR). 4a ed. Porto Alegre: Artmed; 2002. p. 59-69.

23. Del-Ben CM, Vilela JAA, Crippa JAS, Hallak JEC, Labate CM, Zuardi A. Confiabilidade da "Entrevista Clínica Estruturada para o DSM-IV - Versão Clínica" traduzida para 0 português. Rev Bras Psiquiatr. 2001;23:156-9.

24. Corrigan PW. Toolkit for Evaluating Programs Meant to Erase the stigma of Mental IIIness; Illinois Institute of Technology. 2008.

25. Fleck M, Louzada S, Xavier M, Chachamovich E, Vieira G, Santos L, et al. Aplicação da versão em português do instrumento abreviado de avaliação de qualidade de vida "WHOQOLbref". Rev Saúde Pública. 2000;34(2):178-83.

26. Martini LC, Attux C, Bressan RA, Mari JJ. Adaptação cultural, validade e confiabilidade da versão brasileira do Inventário de Habilidades de Vida Independente: versão do paciente (ILSS-BR/P), na esquizofrenia. Rev Psiquiatr Clín. 2012;39(1):12-8.

27. Chaves GFS, Oliveira A0, Forlenza V, Nunes, PV. Escalas de avaliação para Terapia Ocupacional no Brasil. Rev Ter Ocup Univ. São Paulo. 2010;21(3):240-6.

28. Kay SR, Fiszbein A, Opler LA. The positive and negative syndrome scale (PANSS) for schizophrenia. Schizophr Bull. 1987;13(2):261-76.

29. American Psychiatric Association. Avaliação multiaxial. In: Manual diagnóstico e estatístico de transtornos mentais (DSM-IV-TR). 4ª ed. Porto Alegre: Artmed; 2002. p. 59-69.

30. Lima MS, Soares GBO, Paoliello G, Vieira RM, Martins CM, Mota Jl, et al. Versão em português da Impressão Clínica Global - Escala de Esquizofrenia: estudo de validação. Rev Bras Psiquiatria. 2007;29(3):246-9.

31. Bressan RA, Chaves AC, Shirakawa I, de Mari J. Validity study of the Brazilian version of the Calgary Depression Scale for Schizophrenia. Schizophr Res. 1998;32(1):41-9.

32. Young SL, Ensing DS. Exploring recovery from the perspective of people with psychiatric disabilities. Psychiatr Rehabil J. 1999;22(3):219-31.

33. Deegan PE. Recovery, uma viagem do coração. In: Ornelas J (ed.). Actas - Novos desafios na reabilitação de pessoas com doença mental. Lisboa: Edições AEIPS; 1999.

34. Chamberlin J. The Ex-Patients' Movement: Where We've Been and Where We're Going. J Mind Behav. 1990;11(3-4):323-36.

35. Chamberlin J. A defesa dos direitos das pessoas com doença mental: Desenvolvimento de uma perspectiva de direitos humanos. Lisboa: Edições AEIPS. 2005. p. 45-56.

36. Miranda APM, Villares CC, Pimentel FA. Anjos de uma asa só: processos de superação na esquizofrenia em um grupo de ajuda mútua. Nova Perspectiva Sistêmica. 2014;48:64-79.

37. Houaiss IA. Dicionário eletrônico da língua portuguesa; versão 3.0; Editora Objetiva; 2009. 